\title{
Quantum Mechanical Study of Protonation of Oxygen Ligands in the Laccase Active Site Based on X-Ray Structures of Subatomic Resolution
}

S. Gavryushov, ${ }^{a, c^{*}}$ N. N. Kuzmich, ${ }^{\text {b,c }}$ K. M. Polyakov ${ }^{\text {a }}$

${ }^{a}$ Engelhardt Institute of Molecular Biology, Russian Academy of Sciences, Vavilova str. 32, Moscow, 119991, Russian Federation

${ }^{\mathrm{b}}$ Smorodintsev Research Institute of Influenza, WHO National Influenza Centre of Russia, 15/17 Professor Popov str., Saint-Petersburg, 197376, Russian Federation

${ }^{c}$ Sechenov First Moscow State Medical University, 2-4 Bolshaya Pirogovskaya str., Moscow, 119991, Russian Federation

${ }^{*}$ Correspondence e-mail: sergei_gavryushov@yahoo.com

Keywords: laccase; serial X-ray data; enzymatic oxygen reduction; reaction mechanism; quantum mechanical DFT calculations; oxygen ligands protonation 


\begin{abstract}
Laccases are enzymes catalyzing oxidation of a wide range of organic and inorganic substrates accompanied by molecular oxygen reduction to water. Previously studies of oxygen reduction by laccases have recently been reported. They were based on single-crystal serial X-ray crystallography with increasing absorption doses at subatomic resolution, As a result, coordinates of all non-hydrogen atoms of the active site have been determined with high precision for both oxidized and reduced states of the enzyme. Those data can be used to clarify the mechanism of molecular oxygen reduction by laccases. However, the X-ray data lack information about protonation states of the oxygen ligands involved. Applying quantum mechanical calculations, in the present work protonation of oxygen ligands in the active site of laccase was determined for both reduced and oxidized states of the enzyme (the stable states observed in experiments at reduction of molecular oxygen in laccase). The high precision of Xray-determined atom coordinates allowed us to simplify preliminary calculations of molecular mechanics for models used in the quantum mechanical calculations.
\end{abstract}




\section{Introduction}

Laccases are multi-copper oxidases which catalyze oxidation of different substrates such as, e.g., $p$-diphenols, simultaneously reducing molecular oxygen to water. Laccases have been found in bacteria, fungi, plants, and insects. Starting from the 1990s, laccases have been studied by spectroscopic methods (Solomon et al., 1996) and then structures of laccases were determined by X-ray crystallography (review of results up to 2015 in (Hakulinen \& Rouvinen, 2015)).

Laccases are globular proteins comprised of two or three domains of cupredoxin fold (Hakulinen \& Rouvinen, 2015). Their active site includes four copper ions arranged in two separated centers: the T1 copper site and the trinuclear copper cluster (TNC). Three types of copper ions (Abriata, 2012) in laccases were well established in earlier spectroscopic studies (Solomon et al., 1996; Cole et al., 1990). The TNC contains a triangle of closely located copper ions including a pair of T3 copper ions coordinated by three histidine residues each and one T2 copper ion coordinated by two histidine residues. Electrons are accepted from substrate at the T1 copper site. Via an electron-transfer chain the electrons are transferred to the TNC where molecular oxygen reduction reaction $\mathrm{O}_{2}+4 \mathrm{e}^{-}+4 \mathrm{H}^{+} \rightarrow 2 \mathrm{H}_{2} \mathrm{O}$ occurs (Solomon et al., 1996; Jones and Solomon, 2015). The TNC is connected to surrounding solvent via the T3 and T2 channels clearly observed in structures as chains of water molecules (Fig 1 in Polyakov et al., 2017). Molecular oxygen gets to the TNC through the T3 channel.

The molecular oxygen reduction at the TNC was considered in works by Solomon and coworkers (Cole et al., 1990; Jones and Solomon, 2015) based on spectroscopy and early X-ray studies of ascorbat oxidase (Messerschmidt et al., 1992). It includes a couple of two-electron reduction stages. Among the TNC states at the oxygen reduction, the following ones can be distinguished. In the fully reduced state of the TNC all three copper ions are reduced to charge +1 and two oxygen ligands are involved (one between two T3 copper ions outside TNC and other one near the T2 copper ion in channel T2). The peroxy intermediate (PI) state includes deprotonated hydrogen peroxide located inside the copper ion triangle. The T2 and one of T3 copper ions are oxidized to $\mathrm{Cu}(\mathrm{II})$ and there is oxygen ligand near the T2 copper ion involved. In the native intermediate (NI) state all three copper ions are oxidized to charge $+2 \mathrm{e}$ and there are three oxygen ligands involved. One is the oxygen ligand near ion T2, one in the center of the copper ions triangle and one between two copper ions T3. The last two appear from peroxide when the $\mathrm{O}-\mathrm{O}$ bond is broken. Each of T3 copper ions is five-coordinated by three nitrogen atoms of histidine residues and by two oxygen ligands between the T3 copper ions in the TNC.

The fully reduced and NI states are clearly observed from X-ray data, whereas the PI state has been deduced entirely from spectroscopic data. Features of this oxygen reduction scheme are 
asymmetric location of the two oxygen ligands in respect to copper ions T3 of the TNC in the PI state and involvement of proton transfer between an aspartic acid residue in the T2 channel and the oxygen ligand near the T2 copper ion. It leads to appearance of a hydroxyl ion instead of a water molecule as the T2 copper coordinating oxygen ligand in channel T2 (Augustine et al., 2010). According to experimental data, the fully reduced state is stable, whereas the NI decays but this process is very slow (Lee at al., 2002). It should be noted that the physics of penetration of molecular oxygen into the trinuclear copper cluster has not been discussed within the framework of this scheme.

Based on the abovementioned scheme of molecular oxygen reduction in laccases (Jones and Solomon, 2015), there were done studies using quantum mechanical (QM) and molecular mechanical methods (Rulisek et al., 2005; Yoon and Solomon, 2007; Yoon et al., 2007; Srnec et $a l ., 2011)$. Those QM calculations based on the density functional theory (DFT) agreed with the suggested reaction mechanism and demonstrated a strong dependence of obtained energies on protonation of the oxygen ligands coordinating the TNC copper ions (Yoon et al., 2007; Srnec et $a l ., 2011)$. However, the oxygen ligand transfer from the center of the trinuclear cluster to the copper coordinating position in the T2 channel (Yoon et al., 2007) is questionable. According to high resolution X-ray data structures of laccase (Polyakov et al., 2017), surrounding residues involved into coordination of the TNC copper ions leave no space for such move because of steric restrictions in the trinuclear center.

Recently there have been reported serial structures of laccase with different degree of TNC copper ions reduction solved at high resolution for sets of X-ray data collected from one crystal (Polyakov et al., 2017, Polyakov et al., 2019). These studies as well as other reported results for serial crystallography (Komori et al., 2012; Komori et al. 2014) revealed a picture that contradicted to some extent to the scheme of oxygen reduction suggested earlier. Due to high quality of subatomic resolution data for sets of structures with different degree of oxidation, both oxidized and reduced states of the TNC were resolved and clearly interpreted. As a result, it was possible to determine coordination of the TNC copper ions in the two states (Fig. 1). There were detected two positions of each copper ion T3 corresponding to its states $\mathrm{Cu}(\mathrm{I})$ or $\mathrm{Cu}(\mathrm{II})$. In state $\mathrm{Cu}(\mathrm{I})$ the copper ion is three-coordinated by three histidine residues. In the oxidized state $\mathrm{Cu}(\mathrm{II})$ is five-coordinated by those histidines residues and two oxygen ligands. In the reduced state the distance between the T3 ions is about $5.2 \AA$ and it is about $3.3 \AA$ in the oxidized state. There was detected switching between two positions of the oxygen ligand coordinating the T2 copper ion in channel T2. The latter clearly indicates a change of the T2 copper ion coordination at its oxidation. In the reduced TNC state, $\mathrm{Cu}(\mathrm{I})$ ion $\mathrm{T} 2$ is linearly coordinated to nitrogen atoms of two surrounding histidine residues. The neighboring oxygen ligand in the T2 channel interacts 
with copper ion T2 via electrostatic forces. When the oxygen ligand is found at the center of the trinuclear cluster, the $\mathrm{T} 2 \mathrm{Cu}(\mathrm{II})$ ion has square-planar four-coordination with addition of the oxygen ligand in the T2 channel and this oxygen ligand at the center of the copper ion triangle. As a result, the distance between the $\mathrm{T} 2$ copper ion and the oxygen ligand in the $\mathrm{T} 2$ channel diminishes.

Based on those serial X-ray data, some corrections to the oxygen reduction scheme were discussed (Polyakov et al., 2017). In this model of the reaction, an oxygen molecule, being an induced dipole attracted by the trinuclear cluster of ions $\mathrm{Cu}(\mathrm{I})$, penetrates between two copper ions T3, oxidizing both of then. The appearing PI state is symmetrical in respect to the fivecoordinated T3 ions. It will wait for the next coming electron that reduces one of the T3 copper ions. The following cleavage of the peroxide $\mathrm{O}-\mathrm{O}$ bond is cased by the oxidation of this copper ion and copper ion T2 by the two oxygen atoms and change in coordinating the T3 and T2 copper ions. The final structure corresponds to the NI state of TNC. The next reductions of TNC copper ions due to the electrons supply release two oxygen ligands from the copper ion cluster, restoring the reduced state of TNC. According to this scheme, the change of T2 copper ion coordination is accompanied by the cleavage of the peroxide $\mathrm{O}-\mathrm{O}$ bond. In this scheme all the stable TNC states are quasi symmetrical structures in respect to the pair of copper ions T3, copper ion T2 is oxidized last and there is no need in appearance of a hydroxyl ion in the T2 channel near the T2 copper ion.

The oxidized (NI) and reduced states of the TNC were well interpreted from serial X-ray data. On the other hand, in all structures there were detected no traces of the asymmetrical location of the central oxygen ligand needed for the peroxide intermediate states of the TNC, suggesting for their short life time.

Positions of the TNC atoms and ligands in the oxidized state of the TNC (the native intermediate) are shown in Fig. 1a. Their positions for the TNC reduced state are shown in Fig. 1b. The oxygen ligand in the center of the copper triangle is denoted as W1, the oxygen ligand in channel T2 as W2 and the oxygen ligand in channel T3 as W3. In the reduced state ion $\mathrm{Cu}(\mathrm{I}) \mathrm{T} 2$ is linearly coordinated by two nitrogen atoms of the histidine residues $\mathrm{H} 400$ and H65 and oxygen ligand W2 at its position W2(I) is connected to the ion by only ion-dipole electrostatic interaction, whereas in the fully oxidized state position W2(II) is closer to the ion due to involvement of the oxygen ligand $\mathrm{W} 2$ into the $\mathrm{T} 2 \mathrm{Cu}(\mathrm{II})$ coordination. Positions $\mathrm{Cu} 2(\mathrm{I})$ and $\mathrm{Cu} 2$ (II) almost coincide. 


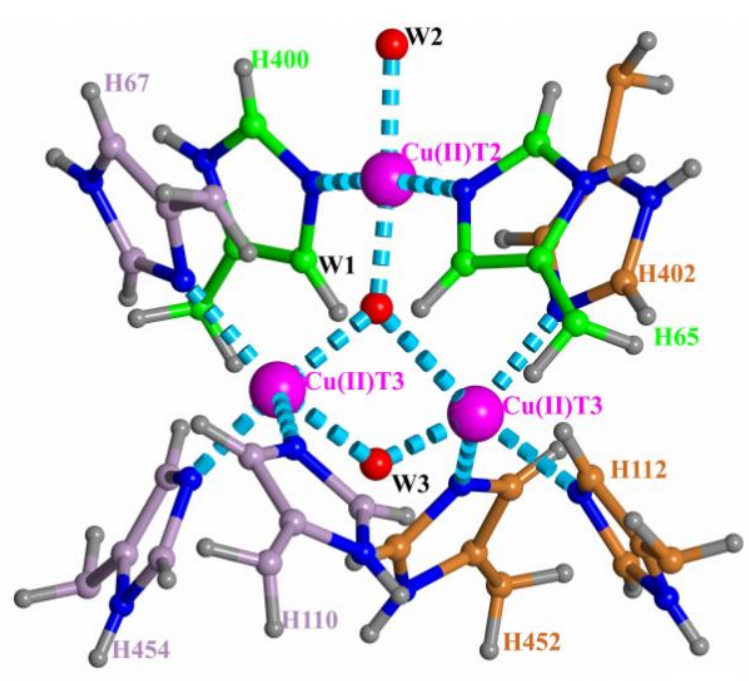

(a)

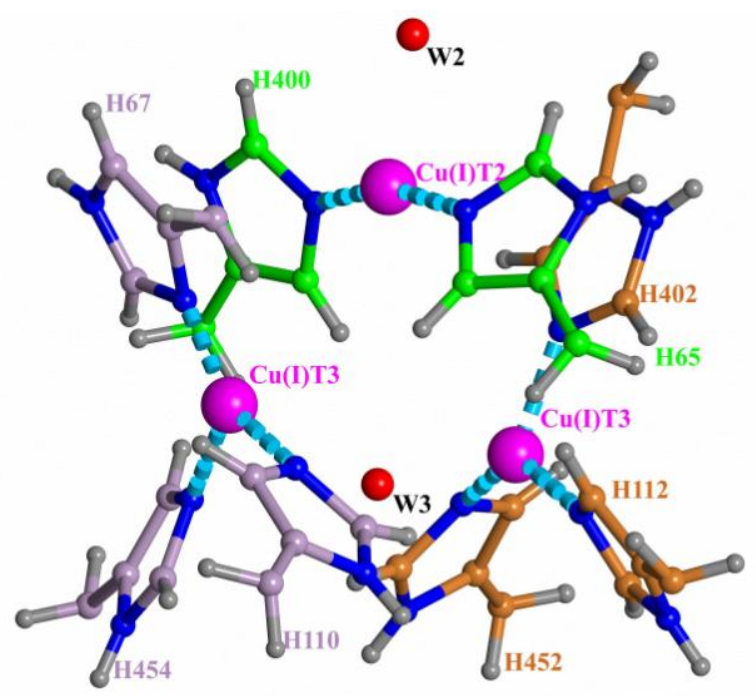

(b)

Figure 1. The oxidized (a) and reduced (b) states of the TNC (Polyakov et al., 2019). Positions of copper ions (purple) are denoted as $\mathrm{Cu}(\mathrm{I}) \mathrm{T} 3$ and $\mathrm{Cu}(\mathrm{II}) \mathrm{T} 3$ for the $\mathrm{T} 3$ ions and $\mathrm{Cu}(\mathrm{I}) \mathrm{T} 2$ / $\mathrm{Cu}(\mathrm{II}) \mathrm{T} 2$ for ion $\mathrm{T} 2$ for the reduced and oxidized states, respectively. There are also shown oxygen ligands (red spheres) and surrounding histidine residues coordinating the three copper ions.

The strong dependence of the QM-calculated energies of states at cleavage of the $\mathrm{O}-\mathrm{O}$ bond upon protonation of the oxygen ion in the center of the trinuclear cluster (Srnec et al., 2011) raises questions about true protonation state of oxygen ligands involved. It cannot be resolved from solution of X-ray data structures where protonation of the oxygen ligands remains undetermined even at atomic resolution. In the present work QM DFT calculations have been applied for a study of protonation of the TNC oxygen ligands (Fig. 1) for the observed stable oxidized (NI) and reduced states of TNC based on serial one-crystal X-ray data at subatomic resolution (Polyakov et al., 2019). QM calculations of energies of the system at different protonation states of oxygen ligands involved allow us to find out preferential states of the complex, which might advance understanding of the molecular mechanism of oxygen reduction in laccases.

\section{Computational Details}

All initial coordinates of non-hydrogen atoms were taken from 6RGP record of Protein Data Bank (Polyakov et al., 2019). The 6RGP structure was solved at subatomic resolution (0.97 $\AA$ ) and presents clearly distinguishable oxidized and reduced enzyme forms (Fig. 1). To model the 
proton transfers, the QM calculations included TNC's adjacent environment in gas phase. Such an approach is possible due to the fact that the TNC is immersed into hydrophobic core of the laccase macromolecule. In all models there were included three TNC copper ions and side chains of eight histidine residues coordinating them.

The chosen molecular model around the active site was a compromise between delivering a minimal adequate chemical environment and computational cost. The input structures were prepared in the following way. Initially the 6RGP structure was imported into Maestro 11.8 graphical interface within Schrodinger 2018-4 software (Schrödinger Release 2018-4, 2018). For oxidized and reduced laccase forms the corresponding copper and oxygen atoms coordinates were chosen from X-ray data according to the analysis performed previously (Polyakov et al., 2019). Then, all hydrogens of the model were added and bond orders were assigned employing the Protein Preparation Wizard and manually edited as necessary for the model chosen. In most cases histidine residues were truncated to their side chains with beta carbons substituted by methyl groups. When proton transfer in the T2 or T3 channels was considered, also the watercoordinating functional groups $(\mathrm{C}=\mathrm{O}, \mathrm{NH}, \mathrm{COO}-)$ were preserved as well as other oxygen ligands. The side chains of amino acids other than histidine were removed and backbone fragments were capped ( $\mathrm{N}$-methylated $\mathrm{C}$-termini and acetylated $\mathrm{N}$-termini). Aspartatic side chains were truncated to acetates. To fill up the free valences where the backbone and side chain fragments were cut off, the hydrogens were added.

All the DFT calculations were performed with Gaussian 16 Revision B 01 (Frisch et al., 2016) software using TPSSh density functional (meta-hybrid density functional: Tao et al., 2003; Staroverov et al., 2003). It performs well in describing reactions of transition metal systems (Jensen, 2008; Moltved and Kepp, 2019). The basis sets were composite: the Pople's splitvalence $3-21 G^{*}$ and $6-31 G^{* *}$ for $p$-elements and effective core potentials (ECP) for copper atoms (Ditchfield et al., 1971; Francl et al., 1982). The pseudopotentials and basis sets themselves were taken for 10 core electrons, multi-electron approximation and completely relativistic (ECP10MDF) (Figgen et al., 2005; Frisch et al., 2016).

Positions of copper ions and most of non-hydrogen atoms of residues were taken from the Xray data structure and kept frozen, whereas all hydrogen atoms, oxygen ligands chosen for a particular model and carbon atoms of capping methyl groups were free. The initial geometry optimization was carried out using smaller $3-21 G^{*}$ basis set for s- and p-elements. There were calculated energy second derivatives for final optimization that was done with larger 6-31G** basis set. Geometry optimizations followed by frequency calculations to confirm the correctness of stationary points found and also thermochemistry calculations including zero-point energy. 
Oxygen ligand protonation was evaluated via comparison of the energies of a system with different protonation of oxygen ligands. The energy barrier of a proton transfer from the W3 to W1 oxygen ligands was also evaluated. The potential barrier at the proton transfer was studied by means of determining the transition state using quadratic synchronous transit 3 (QST3) algorithm (Peng and Schlegel, 1993). The transition state finding was verified by the IRC approach (Fukui, 1981).

\section{Results}

\subsection{Protonation of the W1 oxygen ligand in the center of TNC}

The model for QM DFT calculations to explore protonation of oxygen ligand W1 in the NI state of TNC is shown in Fig. 2. When it is protonated, both oxygen ligands $\mathrm{W} 1$ and $\mathrm{W} 3$ are $^{-} \mathrm{OH}^{-}$ . This model was also applied to the geometry optimizations.

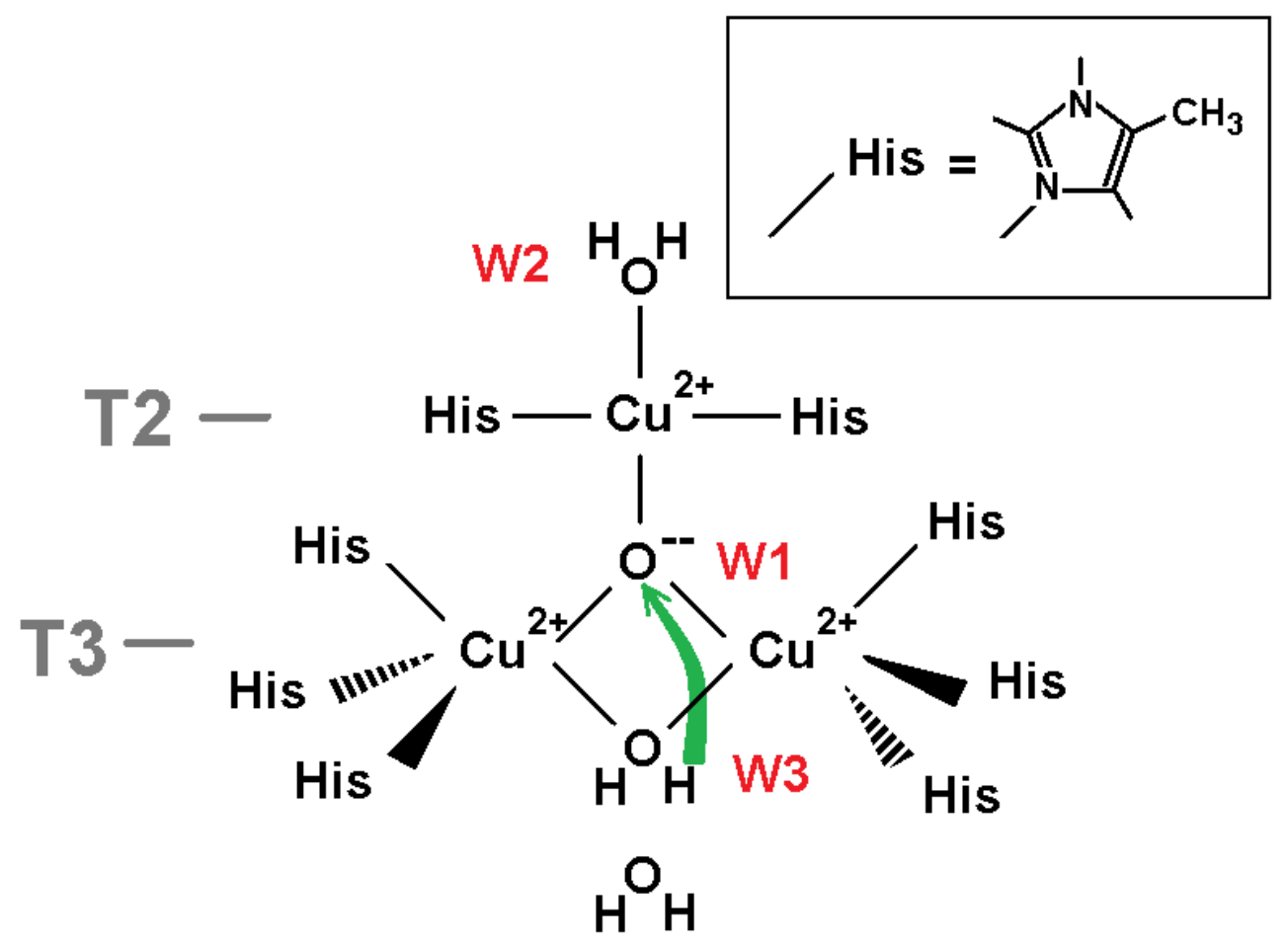

Figure 2. W1 protonation study model. The surroundings of the oxygen ligand W1 in the centre of the copper ions $\mathrm{Cu}$ (II) cluster that were chosen for QM calculations. The green arrow shows the proton transfer in the reaction $\mathrm{H}_{2} \mathrm{O}+\mathrm{O}^{2-} \rightarrow \mathrm{OH}^{-}+\mathrm{OH}^{-}$. Coordinating bonds are depicted as black lines. An oxygen ligand $\left(\mathrm{H}_{2} \mathrm{O}\right)$ in channel $\mathrm{T} 3$ from the structure (pdb entry 6RGP) was included in the model. 
The model includes atoms of eight histidine residues. Residues His 67, His 454, His 110, His 402, His 112, His 452 coordinate the pair of T3 copper ions. Residues His 65 and His 400 coordinate the T2 copper ion. For DFT calculations, the polypeptide chain was truncated and each beta carbon of the residues is replaced by a methyl cap. The model includes three TNC copper ions in the oxidized state $\mathrm{Cu}$ (II) and four oxygen ligands from the $\mathrm{X}$-ray data structure corresponding to the oxidized state of TNC. Three of them are ligands W1, W2 and W3 (Fig. 1a) and the fourth one is oxygen ligand near to W3 in the T3 channel. In all calculations the oxygen atoms of ligands W3 and W2 were retained. Due to steric restrictions inside the trinuclear cluster, two positions of hydrogen of the central $\mathrm{OH}^{-}$ion are only possible: "above" and "below" the plane of copper ions triangle, almost perpendicular to the plane. Both of them have been studied in following geometry optimization and DFT calculations. In the following text they will be called positions "up" and "down". Further in this section we also denote the state of the system according to the protonation state of oxygen ligands $\mathrm{W} 1, \mathrm{~W} 2$, and $\mathrm{W} 3$ as (W1,W2,W3) (for example, $\left(\mathrm{O}^{2-}, \mathrm{H}_{2} \mathrm{O}, \mathrm{H}_{2} \mathrm{O}\right)$ ).

The study included several DFT calculations. Two ones were done with the central oxygen ligand (as $\mathrm{O}^{2-}$ and $\mathrm{OH}^{-}$ion) released to determine its calculated positions. A particular QM study was pointed to determine the transition state at the proton transfer from $\mathrm{H}_{2} \mathrm{O}$ at position $\mathrm{W} 3$ to $\mathrm{O}^{2-}$ at position $\mathrm{W} 1$ in order to evaluate the potential barrier of this reaction. Finally energies of the three states $\left(\left(\mathrm{O}^{2-}, \mathrm{H}_{2} \mathrm{O}, \mathrm{H}_{2} \mathrm{O}\right)\right.$, the transition state, $\left(\mathrm{OH}^{-}\right.$up, $\left.\left.\mathrm{H}_{2} \mathrm{O}, \mathrm{OH}^{-}\right)\right)$were calculated. Energy of state $\left(\mathrm{OH}^{-}\right.$down, $\left.\left.\mathrm{H}_{2} \mathrm{O}, \mathrm{OH}^{-}\right)\right)$was calculated separately.

The QM calculations revealed that $\mathrm{W} 1 \mathrm{O}^{2-}$ ion of the oxidized form should get a proton from oxygen ligand W3 if the latter one is a water molecule. We considered: $(i)$ the coordinate shifts of both protonated $\left(\mathrm{OH}^{-}\right)$and deprotonated $\left(\mathrm{O}^{2-}\right)$ ions in respect to the $\mathrm{T} 2$ copper atom when constraint was removed for W1 oxygen specimen; (ii) energy of proton transfer from W3 water molecule to $\mathrm{W} 1 \mathrm{O}^{2-}$ oxygen ligand. Results of QM calculations are as follows.

(i) The DFT-determined position of W1 ligand was found close to its position in the X-ray data structure. In the case of released protonated W1, the oxygen atom shift relative to the T2 copper ion position accounted for $0.03 \AA$ compared to the input structure from X-ray data, whereas such a shift consisted about $0.12 \AA$ for deprotonated oxygen $\mathrm{O}^{2-}$.

(ii) Using QST3 algorithm (Peng and Schlegel, 1993), there was studied the transition state of the proton transfer from water molecule $\mathrm{W} 3$ to fully deprotonated ion of oxygen $\mathrm{O}^{2-}$ at position W1: $\mathrm{H}_{2} \mathrm{O}+\mathrm{O}^{2-} \rightarrow 2 \mathrm{OH}^{-}$. Results of energy calculations for different states of the system are collected in Table 1. As one can see, the potential barrier is high enough ( $18 \mathrm{kcal} / \mathrm{mole})$, but the final state $\left(\mathrm{OH}^{-}, \mathrm{H}_{2} \mathrm{O}, \mathrm{OH}^{-}\right)$has a noticeable lower energy than the initial state (decreased by 
$-20 \mathrm{kcal} / \mathrm{mole}$ ) and there is a relatively small difference between two states of the hydroxyl ion (hydrogen atom "up" and "down") of about $6 \mathrm{kcal} / \mathrm{mole}$.

It should be noted that we used $\mathrm{H}_{2} \mathrm{O}$ as ligand W2 (Fig. 2), but in a case of $\mathrm{OH}^{-}$instead of $\mathrm{H}_{2} \mathrm{O}$ this energy difference in a favor of a hydroxyl ion at W1 would only be higher, as the proton is additionally attracted by the negatively charged hydroxyl ion at W2.

Table 1. DFT calculations of state energy differences at a proton transfer from oxygen ligand $\mathrm{W} 3\left(\mathrm{H}_{2} \mathrm{O}\right)$ to oxygen ligand $\mathrm{W} 1\left(\mathrm{O}^{2-}\right)$.

\begin{tabular}{|l|c|c|c|c|}
\hline \multicolumn{1}{|c|}{} & \multicolumn{4}{|c|}{$\Delta E=E_{2}-E_{1}, \mathrm{kcal} / \mathrm{mol}$} \\
\hline $\begin{array}{c}\text { state } \\
1\end{array}$ & $\left(\mathrm{O}^{2-}, \mathrm{H}_{2} \mathrm{O}, \mathrm{H}_{2} \mathrm{O}\right)$ & $\begin{array}{l}\text { transition } \\
\text { state }\end{array}$ & $\left(\mathrm{OH}^{-}\right.$up $\left., \mathrm{H}_{2} \mathrm{O}, \mathrm{OH}^{-}\right)$ & $\left(\mathrm{OH}^{-} \text {down, } \mathrm{H}_{2} \mathrm{O}, \mathrm{OH}^{-}\right)^{\mathrm{a}}$ \\
\hline$\left(\mathrm{O}^{2-}, \mathrm{H}_{2} \mathrm{O}, \mathrm{H}_{2} \mathrm{O}\right)$ & - & 18.4 & -27.1 & -21.6 \\
\hline transition state & & - & -45.5 & - \\
\hline$\left(\mathrm{OH}^{-}\right.$up, $\left.\mathrm{H}_{2} \mathrm{O}, \mathrm{OH}^{-}\right)$ & & & - & 5.5 \\
\hline$\left(\mathrm{OH}^{-}\right.$down, $\left.\mathrm{H}_{2} \mathrm{O}_{2} \mathrm{OH}^{-}\right)$ & & & & - \\
\hline
\end{tabular}

${ }^{\mathrm{a}}$ The transition state was not studied for this reaction product.

\subsection{Protonation of $\mathrm{W} 3$ oxygen ligand in the oxidized state of TNC.}

The molecular model for DFT calculations is shown in Fig. 3. Transfer of a proton from the neighboring oxygen ligand in the $\mathrm{T} 3$ channel as a hydroxonium cation to $\mathrm{W} 3 \mathrm{OH}^{-}$was considered. The protonation of oxygen ligand at position W3 is estimated via the difference of DFT-calculated energies of these two states. The input structure in calculation setup comprised T3 channel including acetate fragment of Asp 456, phenol group of Tyr 117 as a hydrogen-bond donor and extended part of His 454 residues. The $\mathrm{O}-\mathrm{H}$ bond lengths in hydroxonium cation were fixed. The model was constructed so as all molecular fragments involved can form hydrogen bonds with water molecules chosen in channel T3 or interact with them via long-range electrostatics. 


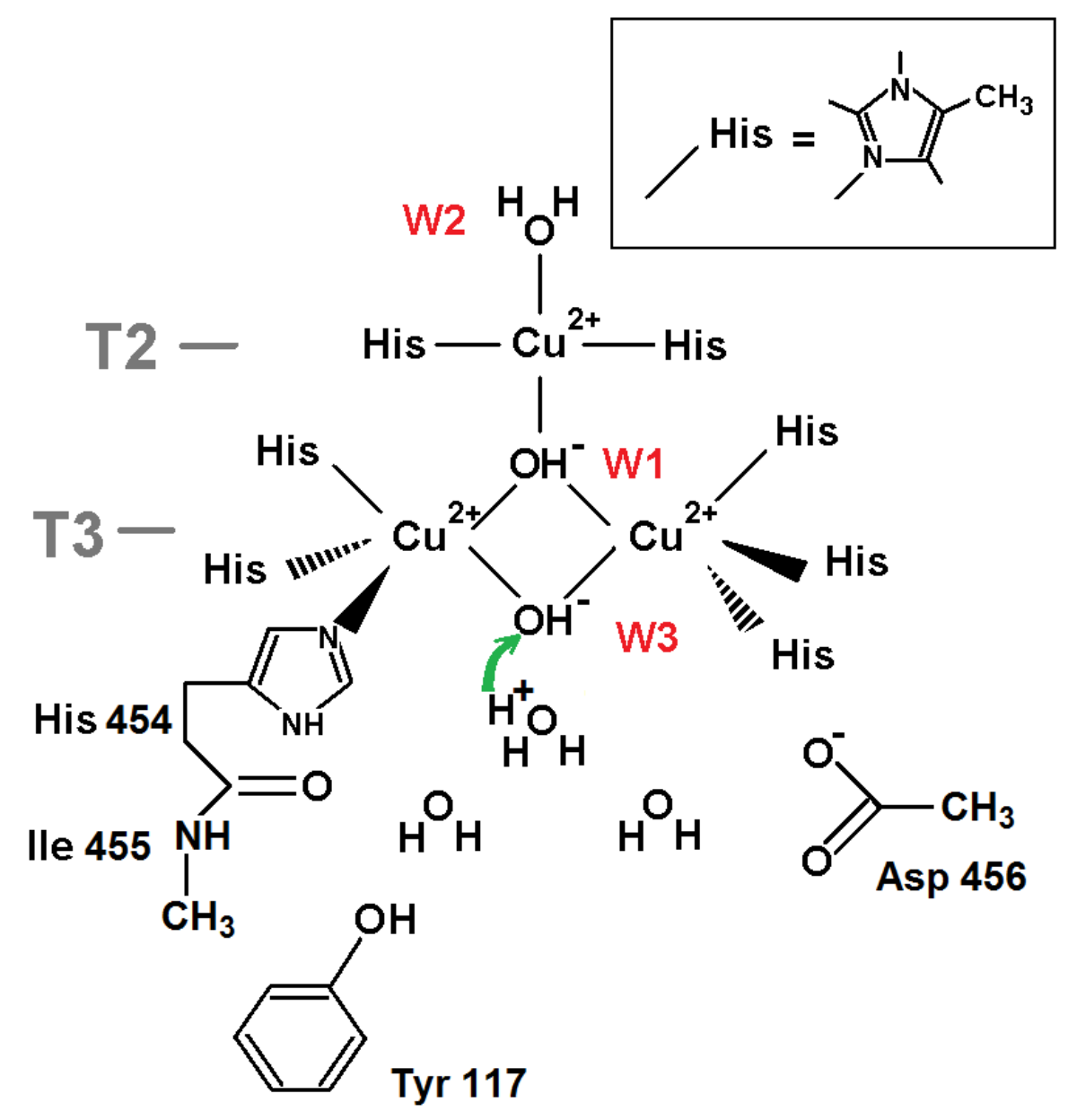

Figure 3. The TNC surroundings for DFT calculations of the proton transfer from hydroxonium cation $\mathrm{H}_{3} \mathrm{O}^{+}$to the neighboring oxygen ligand $\mathrm{W} 3\left(\mathrm{OH}^{-}\right): \mathrm{H}_{3} \mathrm{O}^{+}+\mathrm{OH}^{-} \rightarrow 2 \mathrm{H}_{2} \mathrm{O}$. The central oxygen ligand is presented as a hydroxyl ion $\left(\mathrm{OH}^{-}\right)$. The copper ion coordinating bonds and covalent bonds are depicted as black lines. Three more oxygen ligands in channel T3 in addition to the W3 ligand (pdb entry 6RGP) are added to model.

The DFT calculation results suggest that the system energy grows at the proton transfer from hydroxonium cation located at a position of the next towards T3 channel entrance oxygen atom to the hydroxyl ion at position W3: the energy difference consists $+6.28 \mathrm{kcal} / \mathrm{mol}$. Thus, according to this calculation, oxygen ligand W3 should be treated as $\mathrm{OH}^{-}$. However, it will be $\mathrm{H}_{2} \mathrm{O}$ in reality (see section Discussion).

\subsection{Protonation of oxygen ligand $\mathrm{W} 3$ in the reduced state of $\mathrm{TNC}$.}

The protonation of W3 in the reduced state of TNC (Fig. 1b) was studied via DFT-calculated energies for a system similar to considered for oxidized TNC. The only difference is absence of the $\mathrm{W} 1$ oxygen ligand, all three copper ions are reduced to $\mathrm{Cu}(\mathrm{I})$ and both $\mathrm{W} 2$ and $\mathrm{W} 3$ are 
retained via electrostatic interactions (Fig. 4). In this case we consider two proton transfers in the channel, i.e. energies of three systems are calculated.

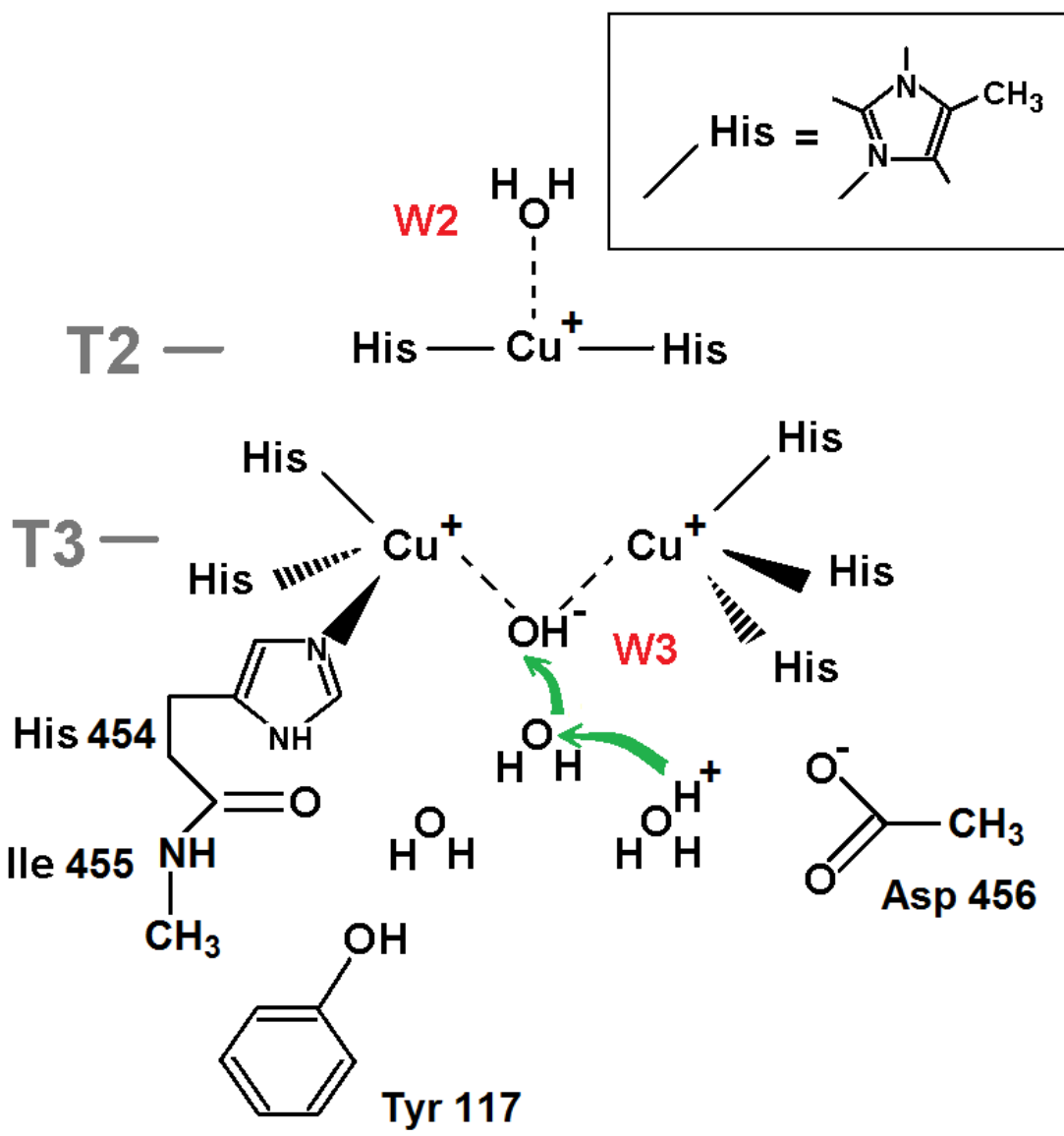

Figure 4. T3 channel environment chosen for DFT calculations of three states to explore protonation of oxygen ligand W3 when the TNC is reduced. The electrostatic interactions are shown as dotted lines. The coordinating and covalent bonds are shown as solid lines.

The DFT-calculated results unambiguously suggest in favor of a water molecule as ligand W3. The energy differences are given in Table 2 where we describe the state as $(\mathrm{W} 3+2, \mathrm{~W} 3+1, \mathrm{~W} 3)$ with $\mathrm{W} 3+1$ and $\mathrm{W} 3+2$ denoting first and second oxygen ligand from the W3 oxygen ligand in the channel, respectively. These results are in accordance with expectations since induced polarization of an oxygen molecule would hardly be competitive with doubled energy of ionic pairs $\mathrm{OH}^{-}-\mathrm{Cu}^{+}$to approach the pair of copper ions T3. On the other hand, competition between an induced dipole of molecular oxygen and a permanent dipole of a water molecule looks plausible enough. 
Table 2. DFT-calculated energy differences for states with different protonation of oxygen ligand W3 and neighboring oxygen ligands at proton transfer shown in Fig. 4.

\begin{tabular}{|c|c|c|c|}
\hline- & \multicolumn{3}{|c|}{$\Delta E=E_{2}-E_{1}, \mathrm{kcal} / \mathrm{mol}$} \\
\hline $\begin{array}{l}2 \\
\text { state } \\
1\end{array}$ & $\left(\mathrm{H}_{3} \mathrm{O}^{+}, \mathrm{H}_{2} \mathrm{O}, \mathrm{OH}^{-}\right)$ & $\left(\mathrm{H}_{2} \mathrm{O}, \mathrm{H}_{3} \mathrm{O}^{+}, \mathrm{OH}^{-}\right)$ & $\left(\mathrm{H}_{2} \mathrm{O}, \mathrm{H}_{2} \mathrm{O}, \mathrm{H}_{2} \mathrm{O}\right)$ \\
\hline$\left(\mathrm{H}_{3} \mathrm{O}^{+}, \mathrm{H}_{2} \mathrm{O}, \mathrm{OH}^{-}\right)$ & - & -13.0 & -31.2 \\
\hline$\left(\mathrm{H}_{2} \mathrm{O}, \mathrm{H}_{3} \mathrm{O}^{+}, \mathrm{OH}^{-}\right)$ & & - & -18.2 \\
\hline$\left(\mathrm{H}_{2} \mathrm{O}, \mathrm{H}_{2} \mathrm{O}, \mathrm{H}_{2} \mathrm{O}\right)$ & & & - \\
\hline
\end{tabular}

\subsection{Protonation of the W2 oxygen ligand in the reduced state of TNC.}

In this section we describe results of DFT calculations for the reduced state of TNC (Fig. 1b). To determine protonation of oxygen ligand W2, energies of states with the W2 oxygen ligand as $\mathrm{H}_{2} \mathrm{O}$ and $\mathrm{OH}^{-}$are compared. The TNC environment chosen as a model of DFT calculations is shown in Fig. 5. Energies of three states of the system are calculated. In this section we will denote each state as $(\mathrm{W} 2+2, \mathrm{~W} 2+1, \mathrm{~W} 2)$ with $\mathrm{W} 2+1$ and $\mathrm{W} 2+2$ denoting first and second oxygen ligand from oxygen ligand W2 in the channel, respectively. Then three states considered will be $\left(\mathrm{H}_{3} \mathrm{O}^{+}, \mathrm{H}_{2} \mathrm{O}, \mathrm{OH}^{-}\right),\left(\mathrm{H}_{2} \mathrm{O}, \mathrm{H}_{3} \mathrm{O}^{+}, \mathrm{OH}^{-}\right)$and $\left(\mathrm{H}_{2} \mathrm{O}, \mathrm{H}_{2} \mathrm{O}, \mathrm{H}_{2} \mathrm{O}\right)$.

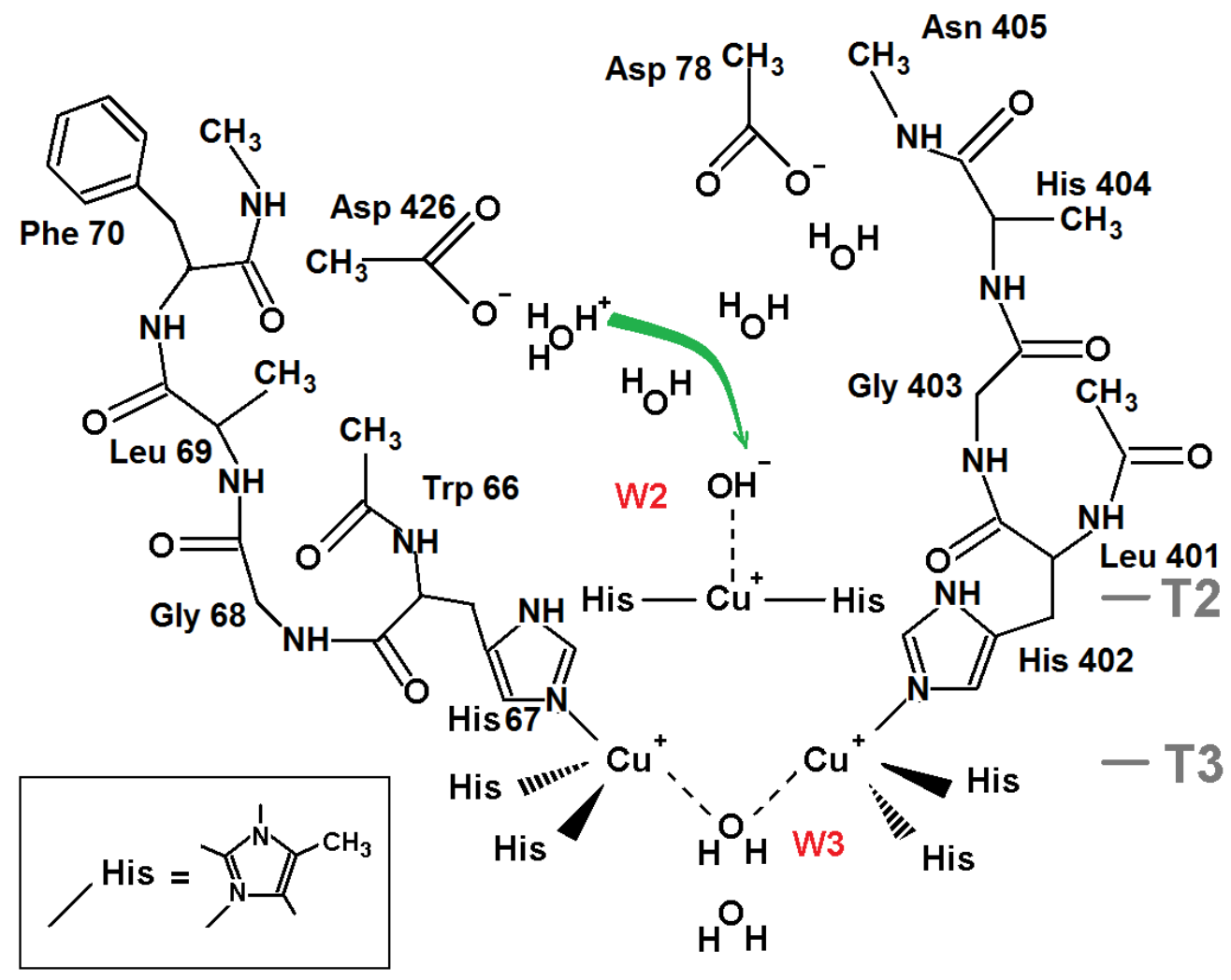


Figure 5. DFT calculations model chosen for a proton transfer along the water molecules chain of the T2 channel to $\mathrm{OH}^{-}$at position W2(I) in the reduced state of TNC. Oxygen ligands $\mathrm{H}_{3} \mathrm{O}^{+}$, $\mathrm{H}_{2} \mathrm{O}$, and $\mathrm{OH}^{-}$(W2) correspond to the proton transfer chain (marked by the green arrow), other two oxygen ligands in channel T2 are water molecules (all positions from pdb entry 6RGP). Purely electrostatic interactions are depicted as dashed lines. Coordinating and covalent bonds are shown as solid lines. See text for further details.

The model includes two protein chains surrounding channel T2. Uncharged amino acid side chains are replaced by methyl groups. Residues and backbone atoms involved into forming a net of hydrogen bonds with water molecules in the channel are described in more details. Positions of water molecules are taken from the X-ray data structure. Five water molecules in the T2 channel are included in the model: three ones on the way to the exit from the channel (including ligand W2) and two ones linking the W2 ligand and Asp 78 connected to His 402 by a noncovalent bond (His 402, in return, coordinates one of the T3 copper ions). The last aspartic acid was assumed to play an important role as a proton acceptor in the scheme of oxygen reduction in TNC discussed earlier (Jones and Solomon, 2015). In our QM study we consider just a transfer of a proton in the T2 channel from outside. We compare energy of two states: the second water molecule from the W2 ligand $\left(\mathrm{OH}^{-}\right)$is a hydroxonium cation $\left(\mathrm{H}_{3} \mathrm{O}^{+}\right)$versus two $\mathrm{H}_{2} \mathrm{O}$ molecules as these two ligands. In other words, we consider a proton transfer along the T2 channel to the hydroxyl ion as ligand W2 in the reduced state of TNC. Now the starting position of W2(I) is taken from coordinates interpreted for the reduced TNC (pdb entry 6RGP, Fig. 1b) and ligand W3 is treated as a water molecule (Fig. 5). One more water molecule next to W3 is also included in the model.

There were performed geometry optimizations without any spatial constraints for oxygen ligand $\mathrm{W} 2$ for both states of its protonation $\left(\mathrm{OH}^{-}\right.$and $\left.\mathrm{H}_{2} \mathrm{O}\right)$. As follows from those optimizations, the shift of oxygen atom from its X-ray-determined position towards $\mathrm{Cu}(\mathrm{I})$ ion $\mathrm{T} 2$ is negligible $(0.02 \AA)$, whereas the hydroxyl ion appeared notably closer to the T2 ion (shift of $0.32 \AA$ ), which is beyond statistical uncertainty of the structure solution from X-ray data. Thus, these results of optimizations are in accordance with interpretation of oxygen ligand $\mathrm{W} 2$ as $\mathrm{H}_{2} \mathrm{O}$.

Results of calculations TPSSh/6-31G ${ }^{* *}$ also suggest in favor of a water molecule at position W2(I) for the reduced state of TNC. Indeed, a proton transfer from the third oxygen ligand in the T2 channel to hydroxyl ion at position W2(I) is energetically quite favorable $(46.8 \mathrm{kcal} / \mathrm{mol})$. Results of energy DFT calculations are given in Table 3. It should be noted that replacement of a water molecule as ligand W3 by a hydroxyl ion only increases this difference in favor of $\mathrm{H}_{2} \mathrm{O}$ as the W2 ligand since a hydroxyl ion attracts the transferred proton. 
Table 3. DFT-calculated energy differences for states with different protonation of oxygen ligand W2 and neighboring oxygen ligands at proton transfer shown in Fig. 5.

\begin{tabular}{|c|c|c|c|}
\hline - & \multicolumn{3}{|c|}{$\Delta E=E_{2}-E_{1}, \mathrm{kcal} / \mathrm{mol}$} \\
\hline $\begin{array}{ll} & \\
\text { state } & \\
1 & \end{array}$ & $\left(\mathrm{H}_{3} \mathrm{O}^{+}, \mathrm{H}_{2} \mathrm{O}, \mathrm{OH}^{-}\right)$ & $\left(\mathrm{H}_{2} \mathrm{O}, \mathrm{H}_{3} \mathrm{O}^{+}, \mathrm{OH}^{-}\right)$ & $\left(\mathrm{H}_{2} \mathrm{O}, \mathrm{H}_{2} \mathrm{O}, \mathrm{H}_{2} \mathrm{O}\right)$ \\
\hline$\left(\mathrm{H}_{3} \mathrm{O}^{+}, \mathrm{H}_{2} \mathrm{O}, \mathrm{OH}^{-}\right)$ & - & -5.3 & -46.8 \\
\hline$\left(\mathrm{H}_{2} \mathrm{O}, \mathrm{H}_{3} \mathrm{O}^{+}, \mathrm{OH}^{-}\right)$ & & - & -41.5 \\
\hline$\left(\mathrm{H}_{2} \mathrm{O}, \mathrm{H}_{2} \mathrm{O}, \mathrm{H}_{2} \mathrm{O}\right)$ & & & - \\
\hline
\end{tabular}

\subsection{Protonation of the W2 oxygen for the oxidized state of TNC.}

The model to estimate protonation of the W2 oxygen ligand in the oxidized state of TNC (Fig. 1a) is similar to one discussed in the previous section. The only difference is coordinates of TNC copper ions and oxygen ligands $\mathrm{W} 1, \mathrm{~W} 2$ and $\mathrm{W} 3$ taken from the TNC oxidized state interpreted from X-ray data (pdb entry 6RGP). The model is shown in Fig. 6. Similar to section 3.4 , there were calculated energies of the system at a consequent transfer of a proton from the second water molecule in the T2 channel to a hydroxyl ion at position W2(II). As before, we describe the state as $(\mathrm{W} 2+2, \mathrm{~W} 2+1, \mathrm{~W} 2)$, where $\mathrm{W} 2+2$ is the second oxygen ligand from oxygen ligand $\mathrm{W} 2$ and $\mathrm{W} 2+1$ is the oxygen ligand next to the W2 oxygen ligand in the T2 channel. Results of DFT calculations suggest in favor of a water molecule as the W2 oxygen ligand when the TNC is oxidized. The energy difference between states $\left(\mathrm{H}_{2} \mathrm{O}, \mathrm{H}_{2} \mathrm{O}, \mathrm{H}_{2} \mathrm{O}\right)$ and $\left(\mathrm{H}_{3} \mathrm{O}^{+}, \mathrm{H}_{2} \mathrm{O}, \mathrm{OH}^{-}\right)$ is $-3.8 \mathrm{kcal} / \mathrm{mol}$. Thus, the energy difference for proton transfer to a hydroxyl ion at position W2(II) through two water molecules along the T2 channel is low but still negative. 


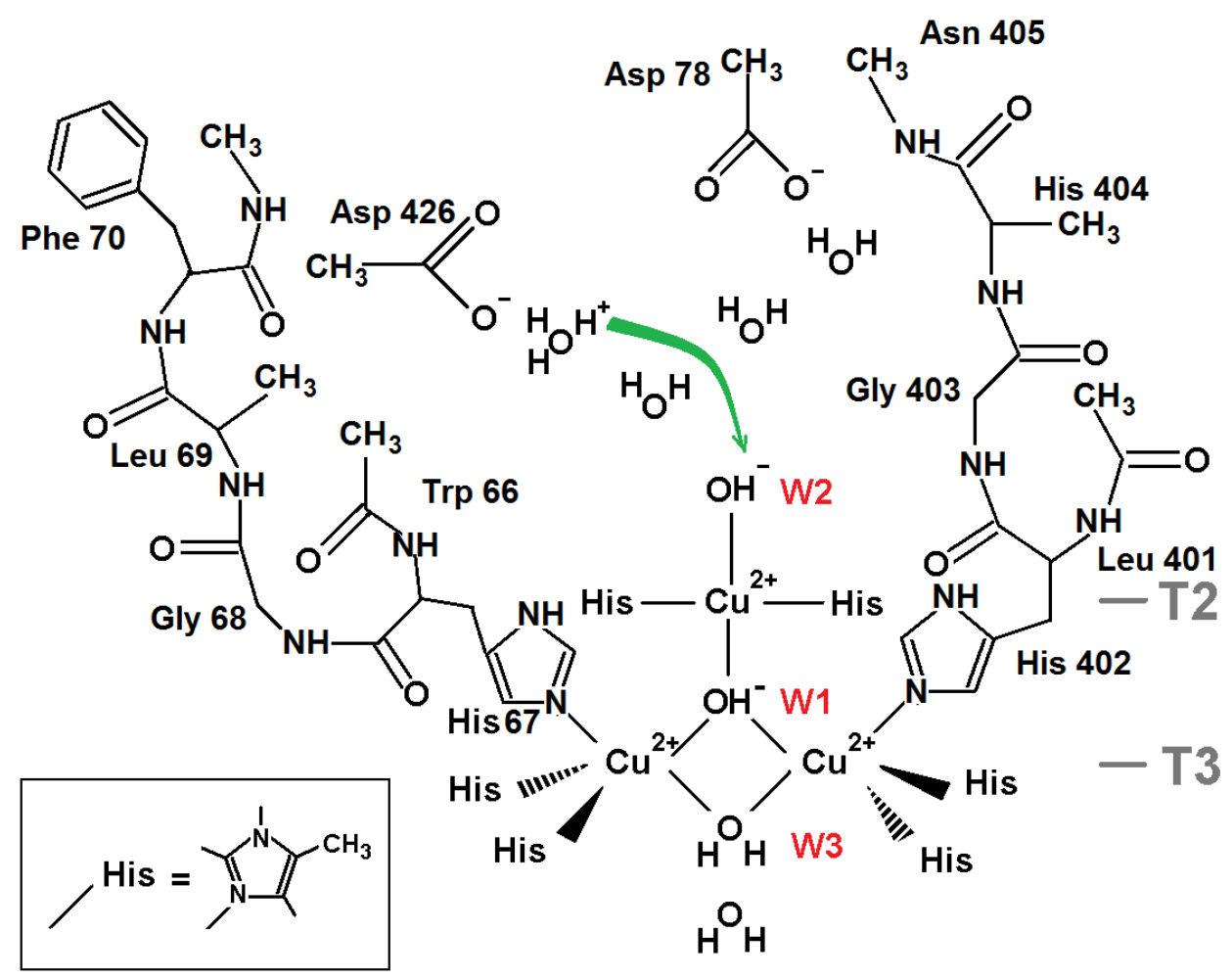

Figure 6. DFT calculations model chosen for a proton transfer along the water molecules chain of the T2 channel to $\mathrm{OH}^{-}$at position W2(II) in the oxidized state of TNC. Oxygen ligands $\mathrm{H}_{3} \mathrm{O}^{+}$, $\mathrm{H}_{2} \mathrm{O}$, and $\mathrm{OH}^{-}$(W2) correspond to the proton transfer chain (marked by the green arrow), other two oxygen ligands are water molecules (all positions from pdb entry 6RGP). Coordinating and covalent bonds are shown as solid lines. See text for further details.

\section{Discussion and conclusions}

The present work is a development of the study of molecular oxygen reduction in laccases (Polyakov et al., 2017; Polyakov et al., 2019). The previous exploration was entirely based on X-ray mono-crystal serial data with increasing absorbed radiation dose at subatomic resolution. Those studies allowed the process of molecular oxygen reduction in TNC to be traced and there were determined the TNC structures in its two stable states (NI and reduced states, Fig. 1). In the present work the protonation of laccase's oxygen ligands in these two stable states is estimated via QM DFT calculations. For the NI state it is shown that oxygen ligand W1 should be a hydroxyl ion, oxygen ligand W2 should be a water molecule, and the preferable form of oxygen ligand W3 should be a water molecule as well, although from the QM calculations it appears to be a hydroxyl ion (see below). For hydroxyl ion at position W1 there are two distinct states that 
differ by location of its hydrogen atom in respect of the three copper ions plane. It was found that the energies of these two states are close to each other (the difference of $\sim 6 \mathrm{kcal} / \mathrm{mol}$ ). A particular study revealed the energy barrier at the W1 oxygen ligand protonation which was found about $20 \mathrm{kcal} / \mathrm{mol}$. For the reduced state of the TNC, oxygen ligand W2 and W3 should be water molecules.

In the present study for QM calculations, coordinates of non-hydrogen atoms were taken from $\mathrm{X}$-ray data solved at subatomic resolution. This allowed us not to perform molecular mechanics optimization and QM optimization for a relatively large system which might bring additional errors. Also, it allowed us to restrain the study by a small number of models without studying various possible placements of the TNC atoms for different states of oxidation as was needed in previous researches (Rulisek et al., 2005).

The QM calculations included TNC's adjacent environment in gas phase. Such an approach is possible due to the fact that the TNC is immersed into hydrophobic core of the laccase macromolecule. An impact of protein's distant atoms was neglected. Only impact of the mean electric field created in TNC area by the charges of the macromolecule's outer parts can be taken into account. But this contribution cannot be high, because a few Angstroms move of a unit charge is only considered. At least it should be much less than a change of the electrostatic potential of a field created by nearby present three copper ions, hydroxyl ions and a couple of charged groups in the channels.

In the QM calculations only transfers of a proton from neighboring water molecules to oxygen ligand (W1, W2 and W3) are considered as the crucial contribution into an estimation of unknown protonation of the ligands. Indeed, the true energy of protonation of the oxygen ligands should be calculated with inclusion of proton migration along the entire channels from the bulk to the TNC oxygen ligands. Such a QM task is beyond our reach. But we do not need to solve this problem. For both channels water molecules form a continuous chain connected by hydrogen bonds. It means that proton can be transferred between neighboring water molecules easily. For channel T3 we just know that protons are transferred along the channel, as they are required for the reaction of the molecular oxygen reduction in the TNC. The main contributor of the protonation of ligands W2 or W3 is expected to be the last moves of protons in the strong repulsive field of the TNC copper cations that has been considered in the present models.

At this point, it should be mentioned that even if the errors of the full proton transfer count up to several $k T$, the conclusions of this protonation study would not be changed. The occupancies of protonated ligands appears as $N_{\mathrm{HOH}} / N_{\mathrm{OH}}=\left(N_{\mathrm{HOH}}{ }^{\mathrm{B}} / N_{\mathrm{OH}}{ }^{\mathrm{B}}\right) \exp \left(-\Delta E_{\mathrm{P}} / k T\right)$, where $N_{\mathrm{HOH}}{ }^{\mathrm{B}}$ and $N_{\mathrm{OH}}{ }^{\mathrm{B}}$ are numbers of water molecules and hydroxyl ions in the bulk solvent outside the protein and $\Delta E_{\mathrm{P}}$ is the thermodynamically averaged work of proton transfer from the bulk to the 
protonated ligand. Assuming the biggest part of this work as the change of the system energy studied in models considered, we obtain for protonation of, e.g., W3 in the oxidized state of TNC (section 3.2) that $\exp \left(-\Delta E_{\mathrm{P}} / k T\right)$ will be $2.5 \cdot 10^{-5}$ at $T=298 \mathrm{~K}$ (that corresponds to $\Delta E_{\mathrm{P}}=6.3$ $\mathrm{kcal} / \mathrm{mol}$ ). On the other hand, even at $\mathrm{pH}=7$, the multiplier $N_{\mathrm{HOH}}{ }^{\mathrm{B}} / N_{\mathrm{OH}}{ }^{\mathrm{B}}$ is $10^{7}$ and it will be higher in acidic environment where fungal laccases function. Therefore, even in this case one should expect rather a water molecule as ligand W3 than a hydroxyl ion there. In all other cases of ligands W2 and W3 studied we should find water molecules at these places.

The reported results are in accordance with the scheme of the molecular oxygen reduction discussed (Polyakov et al., 2017). That scheme was entirely deduced from interpretation of serial $\mathrm{X}$-ray data. In the present work we have confirmed the suggested in the scheme protonation of oxygen ligands W1, W2, and W3 in the NI and reduced state of the TNC determined from X-ray data. Finding the preferable form of the W2 ligand as a water molecule for the oxidized NI state confirms the scheme (Polyakov et al., 2017) and contradicts to the previously discussed scheme (Jones and Solomon, 2015). Anyhow, the intermediate and short-living PI state and the cleavage of the peroxide covalent bond require further QM studies.

\section{Acknowledgments}

This research was supported by RFBR grant 20-04-00683. The computation resources of the Sechenov First Moscow State Medical University and Moscow Joint Supercomputer Center (JSCC) of Russian Academy of Sciences are gratefully acknowledged. 


\section{References}

Abriata, L. A. (2012). Acta Cryst. D68, 1223-1231.

Augustine, A. J., Kjaergaard, C., Qayyum, M., Ziegler, L., Kosman, D. J., Hodgson, K. O., Hedman, B.; Solomon, E. I. (2010). Systematic Perturbation of the Trinuclear Copper Cluster in the Multicopper Oxidases: The Role of Active Site Asymmetry in Its Reduction of $\mathrm{O}_{2}$ to $\mathrm{H}_{2} \mathrm{O} . J$. Am. Chem. Soc. 132, 6057-6067.

Cole, J. L.; Clark, P. A.; Solomon, E. I. (1990). Spectroscopic and chemical studies of the laccase trinuclear copper active site: geometric and electronic structure. J. Am. Chem. Soc. 112, 9534-9548.

Ditchfield, R.; Hehre, W. J.; Pople, J. A. (1971). Self-Consistent Molecular Orbital Methods. IX. An Extended Gaussian-Type Basis for Molecular-Orbital Studies of Organic Molecules. J. Chem. Phys. 54, 724-728.

Figgen, D.; Rauhut, G.; Dolg, M.; Stoll, H. (2005). Energy-consistent pseudopotentials for group 11 and 12 atoms: adjustment to multi-configuration Dirac-Hartree-Fock data. Chem. Phys. 311, 227-244.

Francl, M. M.; Pietro, W. J.; Hehre, W. J.; Binkley, J. S.; DeFrees, D. J.; Pople, J. A.; Gordon, M. S. (1982). Self-Consistent Molecular Orbital Methods. 23. A polarization-type basis set for 2nd-row elements. J. Chem. Phys. 77, 3654-3665.

Frisch, M. J.; Trucks, G. W.; Schlegel, H. B.; Scuseria, G. E.; Robb, M. A.; Cheeseman, J. R.; Scalmani, G.; Barone, V.; Petersson, G. A.; Nakatsuji, H.; Li, X.; Caricato, M.; Marenich, A. V.; Bloino, J.; Janesko, B. G.; Gomperts, R.; Mennucci, B.; Hratchian, H. P.; Ortiz, J. V.; Izmaylov, A. F.; Sonnenberg, J. L.; Williams-Young, D.; Ding, F.; Lipparini, F.; Egidi, F.; Goings, J.; Peng, B.; Petrone, A.; Henderson, T.; Ranasinghe, D.; Zakrzewski, V. G.; Gao, J.; Rega, N.; Zheng, G.; Liang, W.; Hada, M.; Ehara, M.; Toyota, K.; Fukuda, R.; Hasegawa, J.; Ishida, M.; Nakajima, T.; Honda, Y.; Kitao, O.; Nakai, H.; Vreven, T.; Throssell, K.; Montgomery, J. A., Jr.; Peralta, J. E.; Ogliaro, F.; Bearpark, M. J.; Heyd, J. J.; Brothers, E. N.; Kudin, K. N.; Staroverov, V. N.; Keith, T. A.; Kobayashi, R.; Normand, J.; Raghavachari, K.; Rendell, A. P.; Burant, J. C.; 
Iyengar, S. S.; Tomasi, J.; Cossi, M.; Millam, J. M.; Klene, M.; Adamo, C.; Cammi, R.; Ochterski, J. W.; Martin, R. L.; Morokuma, K.; Farkas, O.; Foresman, J. B.; Fox, D. J. (2016). Gaussian 16, Revision B.01, Gaussian, Inc., Wallingford CT.

Fukui, K. (1981). The path of chemical-reactions - The IRC approach. Acc. Chem. Res. 14, 363368.

Hakulinen, N.; Rouvinen, J. (2015). Three-dimensional structures of laccases Cell. Mol. Life Sci. 72, 857-868.

Jensen, K. P. (2008). Bioinorganic Chemistry Modeled with the TPSSh Density Functional. Inorg. Chem. 47, 10357-10365.

Jones, S. M.; Solomon, E. I. (2015). Electron transfer and reaction mechanism of laccases. Cell. Mol. Life Sci. 72, 869-883.

Komori, H.; Sugiyama, R.; Kataoka, K.; Higuchi, Y.; Sakurai, T. (2012). An O-Centered Structure of the Trinuclear Copper Center in the Cys500Ser/Glu506Gln Mutant of CueO and Structural Changes in Low to High X-Ray Dose Conditions. Angew. Chem. Int. Ed. 51, 18611864.

Komori, H.; Sugiyama, R.; Kataoka, K.; Miyazaki, K.; Higuchi, Y.; Sakurai, T. (2014). New insights into the catalytic active-site structure of multicopper oxidases. Acta Cryst. D70, 772779.

Lee, S.-K.; George, S. D.; Antholine, W. E.; Hedman, B.; Hodgson, K. O.; Solomon, E. I. (2002). Nature of the intermediate formed in the reduction of $\mathrm{O}(2)$ to $\mathrm{H}(2) \mathrm{O}$ at the trinuclear copper cluster active site in native laccase. J. Am. Chem. Soc. 124, 6180-6193.

Messerschmidt, A.; Ladenstein, R.; Huber, R.; Bolognesi, M.; Avigliano, L.; Petruzzelli, R.; Rossi, A.; Finazzi-Agry, A. (1992). Refined crystal structure of ascorbate oxidase at $1.9 \AA$ resolution. J. Mol. Biol. 224, 179-205.

Moltved, K. A.; Kepp, K. P. (2019). Performance of Density Functional Theory for Transition Metal Oxygen Bonds. Chem. Phys. Chem. 20, 3210-3220. 
Peng, C.; Schlegel, H. B. (1993). Combining Synchronous Transit and Quasi-Newton Methods for Finding Transition States. Israel J. Chem. 33, 449-454.

Polyakov, K. M.; Gavryushov, S.; Ivanova, S.; Fedorova, T. V.; Glazunova, O. A.; Popov, A. N.; Koroleva, O. V. (2017). Structural study of the X-ray-induced enzymatic reduction of molecular oxygen to water by Steccherinum murashkinskyi laccase: insights into the reaction mechanism. Acta Cryst. D73, 388-401.

Polyakov, K. M.; Gavryushov, S.; Fedorova, T. V.; Glazunova, O. A.; Popov, A. N. (2019). The subatomic resolution study of laccase inhibition by chloride and fluoride anions using singlecrystal serial crystallography: insights into the enzymatic reaction mechanism. Acta Cryst. D75, 804-816.

Rulisek, L.; Solomon, E. I.; Ryde, U. (2005). A Combined Quantum and Molecular Mechanical Study of the $\mathrm{O}_{2}$ Reductive Cleavage in the Catalytic Cycle of Multicopper Oxidases. Inorg. Chem., 44, 5612-5628.

Schrödinger Release 2018-4: Maestro-Desmond Interoperability Tools. Schrödinger, New York, NY, 2018.

Srnec, M., Ryde, U.; Rulisek, L. (2011). Reductive cleavage of the O-O bond in multicopper oxidases: a QM/MM and QM study. Faraday Discuss. 148, 41-53.

Solomon, E. I.; Sundaram, U. M.; Machonkin, T. E. (1996). Multicopper oxidases and oxygenases. Chem. Rev. 96, 2563-2606.

Staroverov, V. N.; Scuseria, G. E.; Tao, J.; Perdew, J. P. (2003). Comparative assessment of a new nonempirical density functional: Molecules and hydrogen-bonded complexes. J. Chem. Phys. 119, 12129. [Erratum] (2004), 121, 11507(E).

Tao, J. M.; Perdew, J. P.; Staroverov, V. N.; Scuseria, G. E. (2003). Climbing the density functional ladder: Nonempirical meta-generalized gradient approximation designed for molecules and solids. Phys. Rev. Lett. 91, 146401. 
Yoon, J.; Liboiron, B. D.; Sarangi, R.; Hodgson, K. O.; Hedman, B.; Solomon, E. I. (2007). The two oxidized forms of the trinuclear $\mathrm{Cu}$ cluster in the multicopper oxidases and mechanism for the decay of the native intermediate. Proc. Natl. Acad. Sci. USA 104, 13609-13614.

Yoon J.; Solomon E. I. (2007). Electronic structure of the peroxy intermediate and its correlation to the native intermediate in the multicopper oxidases : insights into the reductive cleavage of the O-O bond. J. Am. Chem. Soc. 129, 13127-13136. 